\title{
On the Tensile Tests of Polyurethane and Its Composites with Carbon Nanotubes
}

\author{
Martin Kuběna, ${ }^{1}$ Marek Eliáš, ${ }^{2}$ Lenka Zajíčková ${ }^{(D)}{ }^{2,3}$ Jan Poduška, ${ }^{1}$ and Tomáš Kruml ${ }^{4}$ \\ ${ }^{1}$ Institute of Physics of Materials, Žižkova 22, Brno, Czech Republic \\ ${ }^{2}$ CEITEC Masaryk University, Kamenice 5, Brno, Czech Republic \\ ${ }^{3}$ Dept. Phys. Electronics, Faculty of Science, Masaryk University, Kotlářská 2, Brno, Czech Republic \\ ${ }^{4}$ CEITEC IPM, Žižkova 22, Brno, Czech Republic \\ Correspondence should be addressed to Lenka Zajíčková; lenkaz@physics.muni.cz
}

Received 3 September 2018; Revised 11 March 2019; Accepted 26 March 2019; Published 19 May 2019

Academic Editor: Ilia Ivanov

Copyright (c) 2019 Martin Kuběna et al. This is an open access article distributed under the Creative Commons Attribution License, which permits unrestricted use, distribution, and reproduction in any medium, provided the original work is properly cited.

\begin{abstract}
Neat polyurethane (PU) specimens and composites of polyurethane with variable amounts of multiwalled carbon nanotubes (MWCNTs) were subjected to tensile tests, stress relaxation tests, and strain rate jumps. Since the already published data about the effect of carbon nanotubes addition to polymer matrix are somewhat contradictory, great care was taken to understand the mechanical properties of neat PU specimens. The studies revealed that the tensile curves of neat PU are substantially influenced by several factors, such as strain rate, age, and thickness of the specimens. The addition of MWCNTs into the PU matrix had a negligible effect on the mechanical properties of composites at low strains $(\varepsilon<0.2)$. With increasing strain, the MWCNTs addition augmented the strength of the composites, and at high local deformations, as in the previously reported indentation experiments, the adhesion strength between functionalized carbon nanotubes and PU matrix substantially influences the mechanical behavior. The material response to the transient mechanical test showed an Arrhenius-type behavior and was analyzed accordingly. The magnitude of the activation volume scaled with the average length of the free segment of the polymer molecules.
\end{abstract}

\section{Introduction}

Polyurethane (PU) and carbon nanotubes (CNTs) are materials with extremely different mechanical properties. The properties of the PU can be tailored to a certain extent by choosing the reactants, their ratio, and the synthesis procedure [1]. Nevertheless, it is a material with low elastic modulus and strength, capable of large deformations. Additionally, PU cannot be used for applications at elevated temperatures. In contrast, CNTs possess an extremely high Young's modulus ( $E=1240 \mathrm{GPa})$ [2], which is three orders of magnitude higher than that of a typical polymer, high theoretical fracture stress, flexibility and stiffness, and thermal stability up to $2800^{\circ} \mathrm{C}$ in vacuum $[3,4]$. Moreover, the thermal conductivity is about twice as high as that of diamond, and the electric current carrying capacity is 1000 times higher than that of copper wires [5].
To take advantage of these exceptional properties of CNTs, attempts have been made to develop composites with nanotubes as reinforcement. Much research has been focused on the development of nanotube-based polymer composites, although metals and ceramics have also been used as the matrix [4]. It is generally accepted that the addition of CNTs in the PU matrix can strongly alter optical and electrical properties as well as thermal stability and thermal conductivity [6]. However, the effect on mechanical properties is not so clear because of several issues in the composite preparation. Above all, the uniformity of the CNT dispersion and the adhesion of the nanotubes to the matrix are critical for the mechanical properties of such composites $[7,8]$. As shown in our previous paper [9], the PU composites filled with multiwalled CNTs (MWCNTs) improved the material hardness compared to the neat PU. The Martens hardness determined from depth-sensing indentation measurements was $23 \pm 1 \mathrm{MPa}$ for the neat $\mathrm{PU}$, whereas the 
value $28 \pm 1 \mathrm{MPa}$ was obtained for the PU filled with pristine MWCNTs. The PU composites filled with modified MWCNTs, either chemically $\mathrm{COOH}$-functionalized MWCNTs or MWCNTs with plasma-induced oxygen functionalities, exhibited even higher hardnesses, 51-91 MPa.

In this work, we performed a series of tensile tests on neat PU and its composites with variable amounts of pristine MWCNTs. The comparison of these results with the previously reported indentation measurements [9] provides a better understanding of the mechanical behavior of such materials. Moreover, we demonstrate that the tensile curves of neat PU are substantially influenced by several factors, such as strain rate, age, and thickness of the specimens. Although these factors are often not discussed or even specified, they should be taken into account when comparing different kinds of PU composites. At last, activation volumes measured by stress relaxation test and strain rate jumps tests are discussed.

\section{Experiment}

2.1. Materials and Specimens. The polyurethane was prepared from polyol (AXAPUR U100X), isocyanate (U7012), and a solvent thinner (U6002), supplied by Colorlak, at a weight ratio of $7: 2: 1$. Multiwalled carbon nanotubes (research grade $315 X(X=0-3))$ for the preparation of the PU/ CNT composites were purchased from Nanocyl. According to the datasheets provided by the supplier, the CNTs were prepared by catalytic chemical vapor deposition and purified to $95 \%$, with the rest being metal oxides. Their average diameter was $9.5 \mathrm{~nm}$, and the length was below $1 \mu \mathrm{m}$. The CNTs were added to the polyol, solvent thinner, and an antistatic agent, AtmerTM 163 (synthetic ethoxylated amine from Croda Polymer Additives), and mixed for 2 hours in a magnetic stirrer. The mixing continued for $15 \mathrm{~min}$ in an ultrasonic bath after the addition of isocyanate, and the sample was then poured into a square container with a glass bottom. The raw product was thus a foil whose thickness was determined by the amount of liquid.

Strips of the material were clamped onto a metallic support, and the tensile specimens were prepared by cutting out material that exceeded the borders of the support. Generally, the specimens were prepared with a thickness of $1 \mathrm{~mm}$. To investigate the effect of specimen thickness on mechanical response, specimens with thicknesses lower than $1 \mathrm{~mm}$ were also prepared. The final tensile specimens had the shape of a flat dog bone with a dimension of the gauge part of $12 \times 6 \mathrm{~mm}$. The regularity of the thickness within the gauge length was checked for each specimen.

2.2. Tensile Tests. The MTS Tytron 250 Microforce testing system with a linear servomotor was used for the tensile tests. This machine is designed for small specimen testing; the maximum applied force is $250 \mathrm{~N}$. The tensile tests were performed at a constant crosshead velocity corresponding to a strain rate of $2.3 \times 10^{-3} \mathrm{~s}^{-1}$. Some tests were conducted with a different strain rate in order to investigate the strain rate sensitivity of the material. Transient mechanical tests were also performed: (i) test with strain rate jumps, i.e., the strain rate was increased or decreased during the test, and (ii) stress relaxation tests. The latter were performed as follows: the specimen was loaded with a constant strain rate up to the chosen strain, the crosshead displacement was kept constant using close-loop control for a period of $30 \mathrm{~s}$, and the decrease in stress with time was measured; finally, the tensile deformation continued with the same strain rate as before the transient.

Force was measured with a precise load cell with a maximum capacity of $250 \mathrm{~N}$. It was not possible to attach an extensometer to the gauge length of the specimens. Therefore, the strain was measured using digital image correlation as follows. The displacement of the crosshead $\Delta x_{\text {ch }}$ was measured using an LVDT sensor. Contrast stains were sprayed on three specimens, the images of the gauge length were recorded during straining, and the engineering strain $e=\Delta L / L_{0}$ was measured by comparing the image of the unloaded sample and that of the deformed sample (Figure 1). It was found that a simple linear relation between $\Delta x_{\mathrm{ch}}$ and $e$ is valid:

$$
e=C \Delta x_{\mathrm{ch}},
$$

where $C$ is the calibration constant. Since all the specimens had the same shape (except for the thickness), $C$ was the same for all of them. Once $C$ was known, the engineering strain was calculated from the measured values of $\Delta x_{\mathrm{ch}}$. True strain $\varepsilon$ and true stress $\sigma$ values were calculated by assuming the constant gauge part volume:

$$
\begin{gathered}
\varepsilon=\ln (1+e), \\
\sigma=\frac{F}{S_{0}}(1+e),
\end{gathered}
$$

where $S_{0}$ is the initial cross section of the gauge part.

\section{Results}

The tensile tests and transient tests of polyurethane without the addition of carbon nanotubes were carried out first in order to understand the behavior of this material and identify factors that influence the reproducibility of the measurements. The results below demonstrate that care must be taken in studying the effect of the addition of carbon nanotubes (or other fillers) because other parameters, except the content or type of nanotubes, must be kept the same.

3.1. Tensile Tests of Neat Polyurethane. The mechanical response of neat PU depends strongly on the applied strain rate (Figure 2). A low strain rate $\left(\dot{\varepsilon}=4.6 \times 10^{-4} \mathrm{~s}^{-1}\right)$ resulted in a continuous viscous deformation at low stresses without any visible yield point. The tensile curves at higher strain rates $\left(\dot{\varepsilon}=2.3 \times 10^{-3} \mathrm{~s}^{-1}\right.$ and $\left.\dot{\varepsilon}=2.3 \times 10^{-2} \mathrm{~s}^{-1}\right)$ exhibited an initial linear stage with a slope of $0.55 \mathrm{GPa}$, corresponding to the expected value of Young's modulus for this material. The yield point also depended strongly on the strain rate. A strain rate of $\dot{\varepsilon}=2.3 \times 10^{-3} \mathrm{~s}^{-1}$ was used systematically in the following tests. 


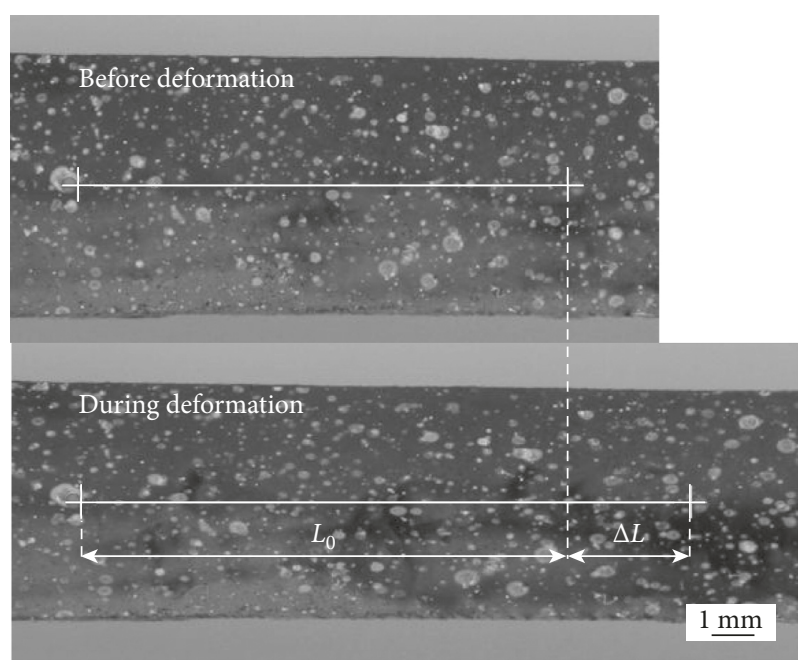

Figure 1: Measurement of strain by image correlation. The distance between the same two objects on the gauge part of the specimen is measured during deformation.

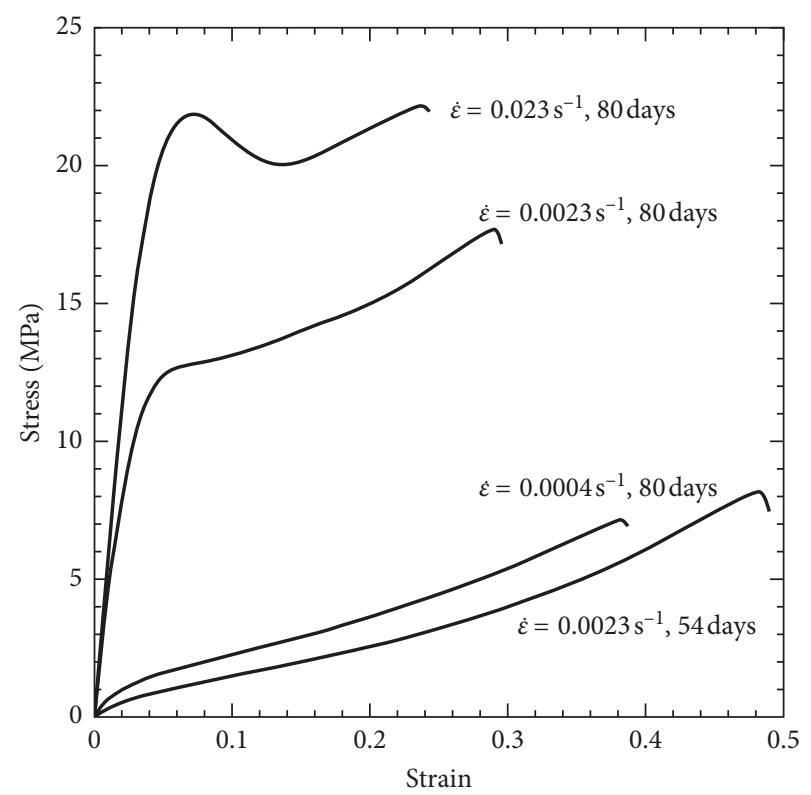

FIGURE 2: Effect of strain rate $\dot{\varepsilon}$ and the age of the specimens on the mechanical behavior of neat PU. The specimens were $1 \mathrm{~mm}$ thick.

The mechanical properties of neat PU also changed substantially with aging time as shown in Figure 2 for two differently aged PU specimens. The material became more resistant to deformation with time but the fracture strain decreased. Various thermal treatments of PU were tested in order to shorten the material stabilization. The satisfactorily stabilized response was observed after 24 hours of aging at room temperature followed by 48 hours at $60^{\circ} \mathrm{C}$. The exposure of the specimens to higher temperatures resulted in surface cracking. Since the data presented in this work were obtained on thermally nonstabilized specimens, their aging must be taken into account when comparing the results.

Another parameter influencing the mechanical strength of the PU specimens was the specimen thickness. The current tensile experiments revealed that the thinner specimens required a higher stress for the same deformation (Figure 3). This effect is discussed in Section 4.1.

3.2. Transient Tests of Neat Polyurethane. Transient tests are performed mainly on metals and alloys with the aim to uncover more details about the operating mechanisms of plastic deformation $[10,11]$. These tests consist of a sudden change of the deformation conditions during the tensile (or compression) test such as (i) stress relaxation test; (ii) strain rate jumps; (iii) transient creep test; (iv) strain (or stress) dip test; and (v) fast temperature changes [10, 12]. In this work, the first two tests were applied to the neat PU specimens.

3.2.1. Stress Relaxation Test. In the stress relaxation test, the tensile test is interrupted, and the displacement of the machine-specimen assembly is kept at a constant value. Traditionally, this test is done by switching off the engine of the machine. The decrease in stress with time is recorded. The stress decrease originates from the plastic deformation of the specimen (the machine must behave purely elastically), and the kinetics of this process is analyzed. The shape of the stress-time curve can be often, but not always, described by a logarithmic function [10].

The measurements were performed with a controlled crosshead displacement, which was kept constant using a back loop. At a chosen level of the strain, the stress relaxation tests were carried out for $30 \mathrm{~s}$. After this period, the tensile test continued at the same strain rate as that before the relaxation. The tensile curves for the five PU specimens at different stages of aging and with different thicknesses are shown in Figure 4 . The drops in stress on the curves corresponded to the stress relaxation tests.

Two examples of stress-time dependency during the stress relaxation tests are shown in Figure 5. It is important to note that the shape of these relaxation curves perfectly follows the logarithmic function. In this case, the thermal activation parameters, especially the apparent activation volume, can be determined as discussed later [10].

3.2.2. Strain Rate Jumps. The apparent activation volume can be measured also by the strain rate jumps. In this case the tensile test is performed with a sudden increase or decrease of the strain rate. Several such experiments were performed on the neat PU specimens. Two typical examples of the material response to the sudden increase or decrease of the strain rate are shown in Figure 6. The definition of stress change induced by the strain rate jump, $\Delta \sigma$, is depicted in both figures. The data are analyzed in the Discussion section.

3.3. Effect of CNT Addition. The specimens of PU filled with different amounts of carbon nanotubes were prepared in order to investigate the effect of CNT addition on mechanical properties. The tensile curves of the PU specimens containing 0, 0.1, 0.5, and $1 \mathrm{wt} . \%$ CNTs are shown in Figure 7. Considering the natural scattering of the data, the mechanical response of all four specimens to the external 


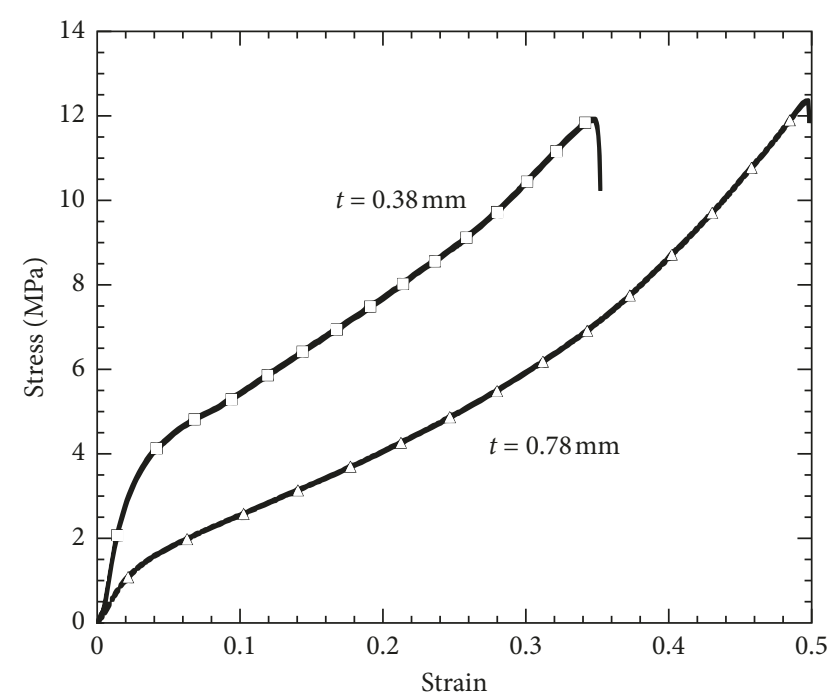

Figure 3: Effect of specimen thickness $t$ for neat PU $\left(\dot{\varepsilon}=2.3 \times 10^{-3} \mathrm{~s}^{-1}\right.$, aged 32 days $)$.

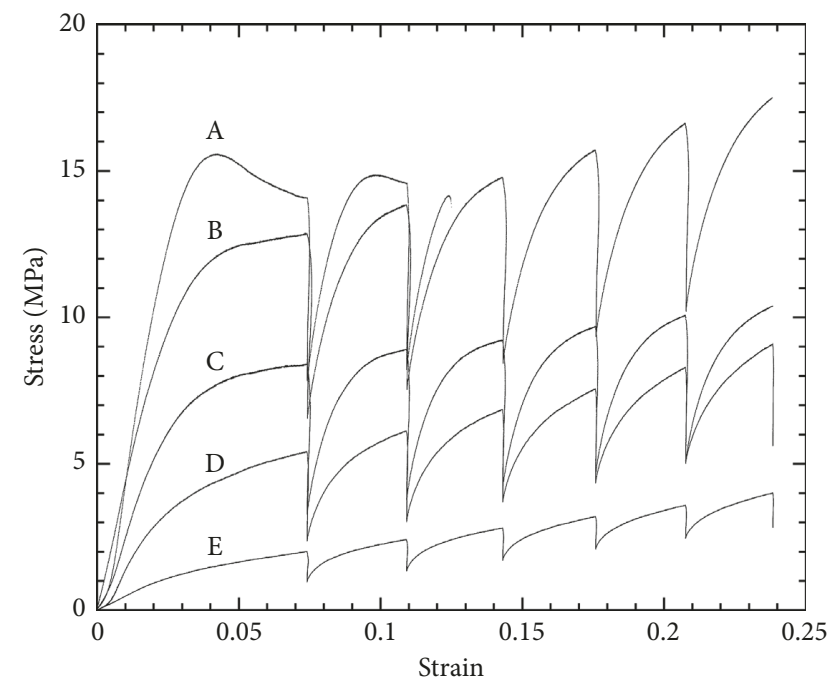

FIgURE 4: Tensile curves of five PU specimens at various stages of aging and with various thicknesses, interrupted by $30 \mathrm{~s}$ stress relaxation tests; $\dot{\varepsilon}=2.3 \times 10^{-3} \mathrm{~s}^{-1}$. A/age of 190 days, $t=0.5 \mathrm{~mm}$; B/ 252 days, $t=1 \mathrm{~mm}$; $\mathrm{C} / 192$ days, $t=0.75 \mathrm{~mm}$; $/ 32$ days, $t=0.5 \mathrm{~mm}$; E/32 days, $t=1 \mathrm{~mm}$.

deformation was the same up to $\sim 0.2$ true strain. The tensile curves of the neat PU and the composite with $0.1 \mathrm{wt} \%$ CNTs overlapped up to fracture. However, the composites with 0.5 and 1 wt.\% CNTs were clearly stronger at higher strains $(\varepsilon>0.2)$. The stresses necessary to deform the $0.5 \mathrm{wt} . \%$ and 1 wt.\% PU/CNT composites to 0.4 true strain were higher than those for the neat PU by $20 \%$ and $33 \%$, respectively. The fracture strain was not influenced by the amount of CNTs and varied from 0.46 to 0.49 .

\section{Discussion}

4.1. Mechanical Behavior of Neat PU. Strong effects of the (i) aging and thermal treatment, (ii) strain rate, and (iii)

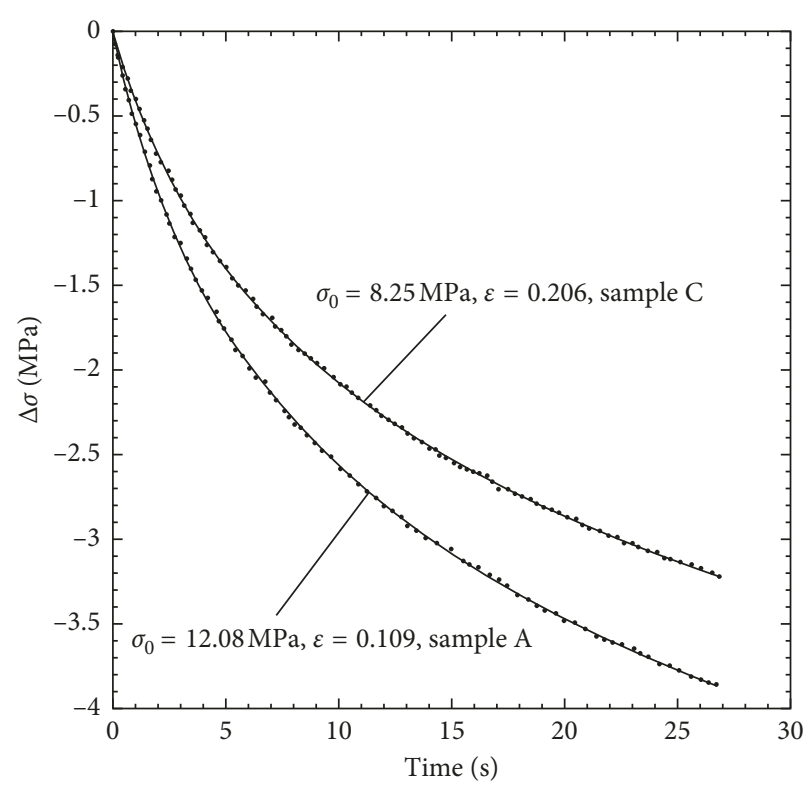

Figure 5: Details of the stress relaxation curves from Fig. 4 for sample $A$ at $\varepsilon=0.109$ and sample $C$ at $\varepsilon=0.206$. The logarithmic fits shown as the solid lines are in excellent agreement with the experimental points (the fitted curve and experimental points overlap).

specimen thickness on the tensile properties are shown in Section 3.1. The increase of the mechanical strength of PU with time after the material preparation can be attributed to the continuous linkage between the polymer chains. The viscoelastic deformation of polymers above the glass transition temperature $T_{\mathrm{g}}$ is time dependent (contrary to the elastic deformation of crystals, which is instantaneous) and is therefore also strain rate dependent.

The effect of PU thickness can be explained by the existence of a mechanically stronger surface layer. Therefore, thinner specimens with a lower amount of weak inner material necessitate a higher stress for the same deformation as shown in Figure 3. The existence of such a layer was noticed by Buršíková et al. [13] who investigated PU specimens of different thicknesses by depth-sensing indentation and explained it by a different solidification processes in the bulk and near the surface, in contact with air. Therefore, care was taken when comparing the results obtained by the depth-sensing microindentation on different PU/CNTs composites [9]. The tensile tests of neat PU carried out in this work led to the similar conclusion, i.e., the surface layers have a higher strength than the inner bulk material. The difference between the PU material near the surface and the bulk PU can be explained by a higher polymer cross linking at the surface.

4.2. Activation Volume of Viscoelastic Deformation. The stress relaxation and strain rate jump tests were developed for measuring the parameters of thermal activation of the plastic deformation of crystals. Occasionally, these tests are also performed on polymers [14-16]. The thermal activation theory $[10,11]$ assumes that there is an energy barrier $\Delta G$ 


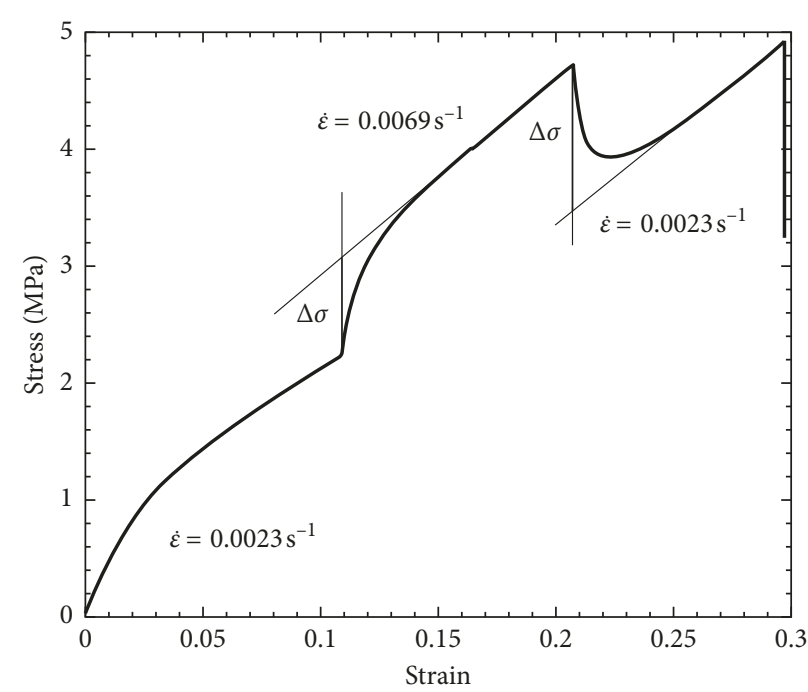

(a)

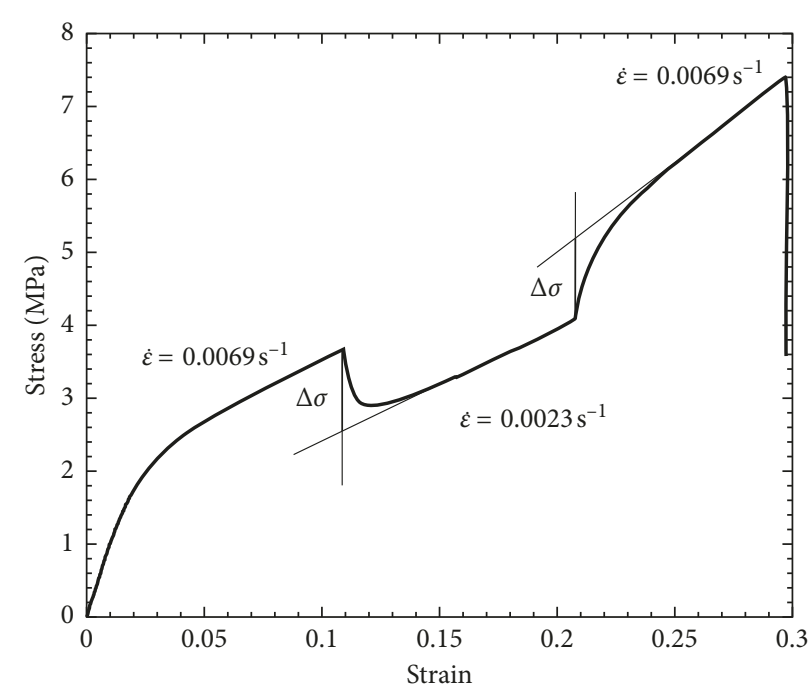

(b)

FIGURE 6: Tensile test with (a) a sudden increase of strain rate from $2.3 \times 10^{-3} \mathrm{~s}^{-1}$ to $6.9 \times 10^{-3} \mathrm{~s}^{-1}$ (age of the specimen was 32 days, $t=1 \mathrm{~mm}$ ) and (b) a sudden decrease of strain rate from $6.9 \times 10^{-3} \mathrm{~s}^{-1}$ to $2.3 \times 10^{-3} \mathrm{~s}^{-1}$ (age of the specimen was 32 days, $t=0.5 \mathrm{~mm}$ ).

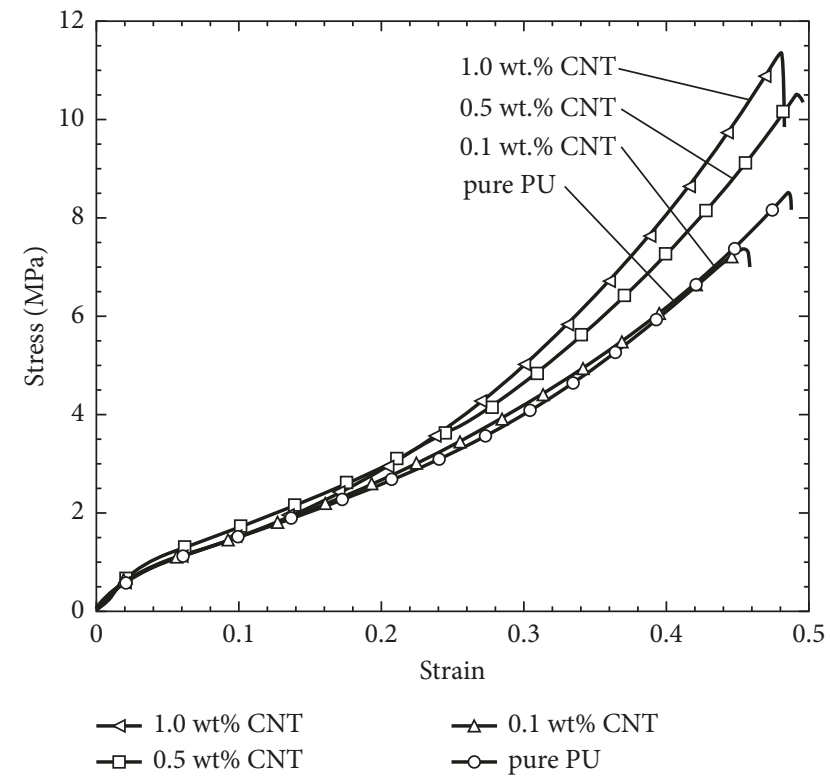

FIgURE 7: Tensile tests of four PU specimens with different contents of carbon nanotubes (strain rate, $2.3 \times 10^{-3} \mathrm{~s}^{-1}$; age of the specimens, 48 days; thickness, $0.65 \mathrm{~mm}$ ).

(activation energy) for plastic deformation, which is overcome with the help of thermal energy in a probabilistic manner. The probability $P$ of the thermally activated event follows an Arrhenius-type equation:

$$
P=P_{0} e^{(-\Delta G / k T)}
$$

where $P_{0}$ is a preexponential factor, $k$ is the Boltzmann constant, and $T$ is the absolute temperature. If stress $\sigma$ is applied, the expression of $\Delta G$ is

$$
\Delta G=\Delta G_{0}-\sigma V
$$

where $\Delta G_{0}$ is the energy barrier at zero stress and $V$ is the parameter with the dimension of volume called the activation volume.

The activation volume can be experimentally measured by transient mechanical tests. It appears that the values measured by the stress relaxation test $\left(V_{\mathrm{r}}\right)$ can differ from those measured by the strain rate jumps $\left(V_{\text {srj }}\right)$. The reasons in the case of dislocation slip driven plasticity have been discussed in previous studies [10, 17].

If the assumptions of the thermal activation theory are correct and equation (3) is valid, the stress relaxation curves $\sigma(t)$ follow a logarithmic formula [10]:

$$
\Delta \sigma=-\frac{k T}{V_{\mathrm{r}}} \ln \left(1+\left(\frac{t}{c}\right)\right),
$$

where $\Delta \sigma=\sigma-\sigma_{0}$ is the stress decrease (negative value) and $t$ is the time variable. There are two free fitting parameters: the time constant $c$ and the apparent activation volume $V_{\mathrm{r}}$. Relaxation curves in Figure 5 are perfectly logarithmic in the whole measured interval. It implies that the attenuation of deformation is governed by the same mechanisms during the whole testing interval.

Another possible method to measure activation volume is the technique of strain rate jumps. In this case, equation (4) can be used [10]:

$$
V_{\text {srj }}=k T \frac{\ln \left(\dot{\varepsilon}_{1} / \dot{\varepsilon}_{2}\right)}{\Delta \sigma},
$$

where $\dot{\varepsilon}_{1}$ and $\dot{\varepsilon}_{2}$ are the strain rates before and after change, respectively, and $\Delta \sigma$ is the corresponding stress change (see Figure 6).

The activation volumes measured by the two techniques are shown in Table 1 and Figure 8. It is visible that the parameter $V_{\text {srj }}$ is systematically lower than $V_{\mathrm{r}}$; however, both parameters follow the same trends. In the case of metals and alloys, both parameters often do not coincide even if the 
TABLE 1: Activation volumes of specimens of neat PU.

\begin{tabular}{lcccccr}
\hline Strain (\%) & 7.4 & 10.9 & $\begin{array}{c}14.3 \\
V_{\mathrm{r}}\left(\mathrm{nm}^{3}\right)\end{array}$ & 17.6 & 20.6 & 10.9 \\
\hline Sample A & 2.74 & 2.75 & & & & \\
Sample B & 2.56 & 2.50 & 2.36 & 2.17 & 2.11 & 0.6 \\
Sample C & 3.61 & 3.42 & 3.31 & 3.17 & 3.03 & 0.94 \\
Sample D & 5.75 & 5.39 & 5.10 & 4.72 & 4.05 & 1.83 \\
Sample E & 19.27 & 18.06 & 16.35 & 15.49 & 14.83 & 4.03 \\
\hline
\end{tabular}

$V_{\mathrm{r}}$ is measured by stress relaxation test and $V_{\text {srj }}$ by strain rate jumps. Specimens are marked as in Figure 4 .

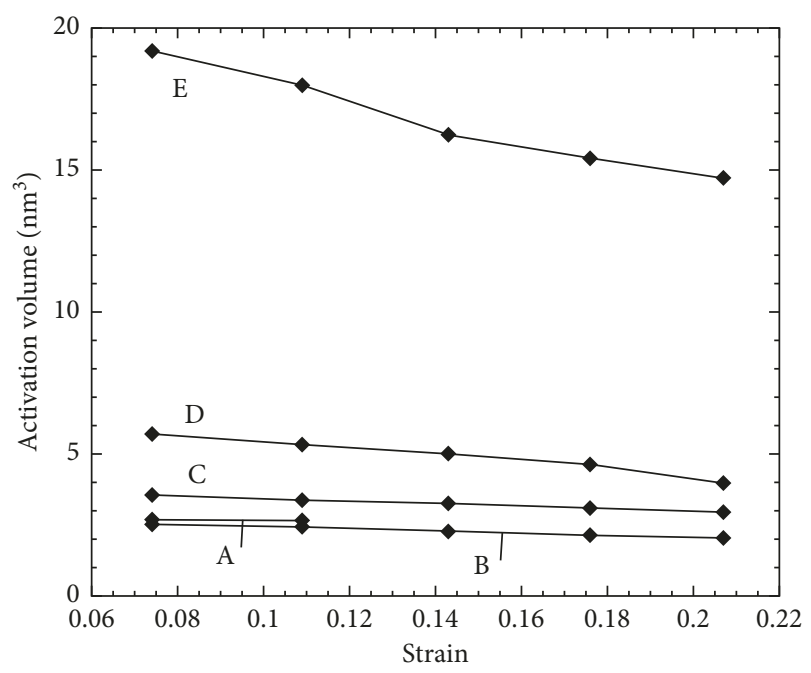

Figure 8: Activation volume $V_{\mathrm{r}}$ measured by the stress relaxation test. A-E correspond to the letters in Figure 4.

difference is usually smaller [10]. Here, the difference is large which implies that the two tests are probably probing different plasticity mechanisms. The experimental material is very sensitive to strain rate (Figure 2) which decreases in stress relaxation test but is constant and higher in strain rate jumps. It can be speculated that when the crosshead is stopped, deformation continues by an easiest mechanisms, e.g., rearrangement of free segments of polymer molecules. However, constant strain rate conditions necessitate also processes at shorter scales (reflected by smaller $V_{\text {srj }}$ ), e.g., breaking the cross links.

Specimens A to E were tested at various states of aging, which is reflected by the different stress-strain curves (Figure 4). It is visible that the weakest specimen (E) shows the largest values of activation volume $\left(V_{\mathrm{r}}\right.$ or $\left.V_{\text {srj }}\right)$ and that the activation volume generally decreases with increasing strength of the PU. The increase of strength with time after material preparation can be interpreted as the continued networking of the polymer and the shortening of the average free segment of the molecules. These results lead to an important conclusion that the activation volume in polymers, like that in crystals, can be a measure of the scale of the activation processes of deformation. The same idea was expressed by Martins and Cruz [18].

The values of $V_{\mathrm{r}}$ varied from $\sim 2$ to $20 \mathrm{~nm}^{3}$, decreased with strain, and were higher for the lower strain rates (Figure 8). The absolute values of $V_{\mathrm{r}}$ were in the same range as that of typical values measured in metals and alloys. The decrease of $V_{\mathrm{r}}$ with deformation is also in agreement with the behavior of metals. In contrast, the metals were much less sensitive to strain rate.

4.3. Effect of Nanotube Addition on Mechanical Properties. Let us first review some published results of the effect of CNT addition on the mechanical properties of polymer composites at temperatures above $T_{\mathrm{g}}$ [4]. Of course, the comparison of published data is complicated because of the differences of the polymer matrix and different CNTs, which may vary in length, diameter, chirality, purity, functionalization, etc. In some papers, the fracture stresses are compared. This is a problematic idea because fracture stress is a parameter with large scatter due to the probabilistic nature of the existence of a large defect in the sample. Therefore, only stress at a given strain (i.e., the proof stress) is compared here. Yao et al. [8] reported an approximately 10\% increase of strength for composites containing $2 \mathrm{wt} . \%$ CNTs. Wang et al. [6] observed the deterioration of elastic modulus and no clear effect of CNT addition on the strength of the composite. Barick and Tripathy [19], in a detailed study, concluded that the properties of the $5 \mathrm{wt}$ \% MWCNT-filled nanocomposites were not significantly enhanced. Other papers [20-22] have reported a small positive effect on elastic modulus and/or strength. In two papers [23, 24], the composites were able to deform to more than $100 \%$. Both papers showed an increased effect of CNT addition with deformation. Xiong et al. observed a low effect of CNT addition up to a strain of $\sim 150 \%$ and an increasing effect at large strain. Finally, in our previous report [9], a substantial effect of CNT addition was observed on indentation properties, including differences in the composites containing commercial CNTs and functionalized CNTs.

All these papers are in fact in agreement with the present results. No effect of the CNTs on the shape of the tensile curve was present up to $\sim 20 \%$ of strain. Between $\varepsilon=20 \%$ and fracture (which appeared at approximately $45 \%$ of strain), the strength of the material increased with the amount of CNTs. The increase in stress for $\varepsilon=45 \%$ was approximately $20 \%$ for the composites with $1 \mathrm{wt} . \%$ CNTs in comparison with the neat PU.

Combining the results of this study and those of the previous paper [9], we can use the following schematics to illustrate the deformation behavior of the polymer composites with CNT reinforcements. At low strains, the macromolecules are straightened without feeling the 
presence of the nanotubes. At higher strains, at approximately $20 \%$ for the studied material (but this value may depend on the properties of both the matrix and CNTs, e.g., on the length of the CNTs), the further straightening of the molecules starts to be opposed by CNTs connecting the polymer segments. Up to a certain deformation, the functionalization of the CNTs does not play a role. However, local deformations and stresses under the tip of the indenter can be very large, and the interfacial adhesion properties between the CNTs and the PU matrix became very important as documented by the significant changes in the mechanical properties of PU/CNT composites with functionalized CNTs [9] (see Introduction).

\section{Conclusions}

The tensile curves of neat PU are substantially influenced by several factors such as strain rate, age, and thickness of the specimens. It needs to be taken into account when comparing different kinds of materials. At low deformation, the effect of CNT addition to PU is not substantial. Considering the price of the CNTs and difficulties to achieve their good dispersion in a polymer matrix, there is no reason to make polymer/CNT composites for improving the mechanical properties in case of expected low deformations. If the strain exceeds $20 \%$ (local or global), the strength of the composite is increased in comparison with the neat $\mathrm{PU}$ and the strengthening effect increases with the strain. The shape of the stress relaxation curves is perfectly logarithmic in all testing conditions. The activation volume can be measured by the stress relaxation tests or strain rate jumps. It seems that different mechanisms are probed at these two tests.

\section{Data Availability}

The data from mechanical tests used to support the findings of this study are included within the article.

\section{Conflicts of Interest}

The authors declare that they have no conflicts of interest.

\section{Acknowledgments}

This research was financially supported by the ESIF, Project ARMADIT (Reg. no. CZ.02.1.01/0.0/0.0/16_025/0007304), and by European Regional Development Fund Project “CEITEC Nano+” (no. CZ.02.1.01/0.0/0.0/16_013/0001728).

\section{References}

[1] C. Prisacariu, Polyurethane Elastomers: from Morphology to Mechanical Aspects, Springer, Berlin, Germany, 2011.

[2] E. Hernández, C. Goze, P. Bernier, and A. Rubio, "Elastic properties of C and Bx Cy Nz composite nanotubes," Physical Review Letters, vol. 80, no. 20, pp. 4502-4505, 1998.

[3] J.-P. Salvetat, J.-M. Bonard, N. H. Thomson et al., "Mechanical properties of carbon nanotubes," Applied Physics A: Materials Science \& Processing, vol. 69, no. 3, pp. 255-260, 1999.

[4] E. T. Thostenson, Z. Ren, and T.-W. Chou, "Advances in the science and technology of carbon nanotubes and their composites: a review," Composites Science and Technology, vol. 61, no. 13, pp. 1899-1912, 2001.

[5] P. G. Collins and P. Avouris, "Nanotubes for electronics," Scientific American, vol. 283, no. 6, pp. 62-69, 2000.

[6] C. Wang, X. Chen, H. Xie, and R. Cheng, "Effects of carbon nanotube diameter and functionality on the properties of soy polyol-based polyurethane," Composites Part A: Applied Science and Manufacturing, vol. 42, no. 11, pp. 1620-1626, 2011.

[7] J.-P. Salvetat, G. Briggs, J.-M. Bonard et al., "Elastic and shear moduli of single-walled carbon nanotube ropes," Physical Review Letters, vol. 82, no. 5, pp. 944-947, 1999.

[8] Z. Yao, D. Wu, C. Chen, and M. Zhang, "Creep behavior of polyurethane nanocomposites with carbon nanotubes," Composites Part A: Applied Science and Manufacturing, vol. 50, no. 03, pp. 65-72, 2013.

[9] L. Zajíčková, M. Eliáš, V. Buršíková et al., "Low pressure plasmachemical processing of multi-walled carbon nanotubes for the production of polyurethane composite films with improved mechanical properties," Thin Solid Films, vol. 538, pp. 7-15, 2013.

[10] D. Caillard and J. L. Martin, Thermally Activated Mechanisms in Crystal Plasticity, Elsevier, Amsterdam, Netherlands, 2003.

[11] J. L. Martin and T. Kruml, "Characterizing thermally activated dislocation mobility," Journal of Alloys and Compounds, vol. 378, no. 1-2, pp. 2-12, 2004.

[12] J. L. Martin, B. Lo Piccolo, T. Kruml, and J. Bonneville, "Characterization of thermally activated dislocation mechanisms using transient tests," Materials Science and Engineering: $A$, vol. 322 , no. 1-2, pp. 118-125, 2002.

[13] V. Buršíková, Z. Kučerová, L. Zajíčková et al., "Measurement of mechanical properties of composite materials," Chemické Listy, vol. 105, pp. 171-174, 2011.

[14] S. Castagnet, "High-temperature mechanical behavior of semi-crystalline polymers and relationship to a rubber-like "relaxed" state," Mechanics of Materials, vol. 41, no. 2, pp. 75-86, 2009.

[15] D. G. Fotheringham and B. W. Cherry, "The role of recovery forces in the deformation of linear polyethylene," Journal of Materials Science, vol. 13, no. 5, pp. 951-964, 1978.

[16] J. Kubát, L.-Å. Nilsson, and M. Rigdahl, "Internal stresses and two-step stress relaxation of polyethylene," Materials Science and Engineering, vol. 61, no. 3, pp. 267-274, 1983.

[17] P. Spätig, J. Bonneville, and J.-L. Martin, "A new method for activation volume measurements: application to $\mathrm{Ni}_{3}(\mathrm{Al}, \mathrm{Hf})$," Materials Science and Engineering: A, vol. 167, no. 1-2, pp. 73-79, 1993.

[18] J. A. Martins and V. S. Cruz, "Flow activation volume of polystyrene/multiwall carbon nanotubes composites," Polymer, vol. 52, no. 22, pp. 5149-5155, 2011.

[19] A. K. Barick and D. K. Tripathy, "Preparation, characterization and properties of acid functionalized multi-walled carbon nanotube reinforced thermoplastic polyurethane nanocomposites," Materials Science and Engineering: $B$, vol. 176, no. 18, pp. 1435-1447, 2011.

[20] X. Jiang, J. Gu, L. Lin, and Y. Zhang, "Investigation on the modification to polyurethane by multi-walled carbon nanotubes," Pigment \& Resin Technology, vol. 40, no. 4, pp. 240-246, 2011.

[21] P. Russo, M. Lavorgna, F. Piscitelli, D. Acierno, and L. Di Maio, "Thermoplastic polyurethane films reinforced with carbon nanotubes: the effect of processing on the structure and mechanical properties," European Polymer Journal, vol. 49, no. 2, pp. 379-388, 2013. 
[22] D. Qian, E. C. Dickey, R. Andrews, and T. Rantell, "Load transfer and deformation mechanisms in carbon nanotubepolystyrene composites," Applied Physics Letters, vol. 76, no. 20 , pp. $2868-2870,2000$.

[23] L. D. Tijing, C.-H. Park, W. L. Choi et al., "Characterization and mechanical performance comparison of multiwalled carbon nanotube/polyurethane composites fabricated by electrospinning and solution casting," Composites Part B: Engineering, vol. 44, no. 1, pp. 613-619, 2013.

[24] J. Xiong, Z. Zheng, X. Qin, M. Li, H. Li, and X. Wang, "The thermal and mechanical properties of a polyurethane/multiwalled carbon nanotube composite," Carbon, vol. 44, no. 13, pp. 2701-2707, 2006. 


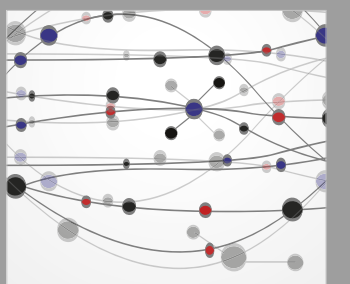

The Scientific World Journal
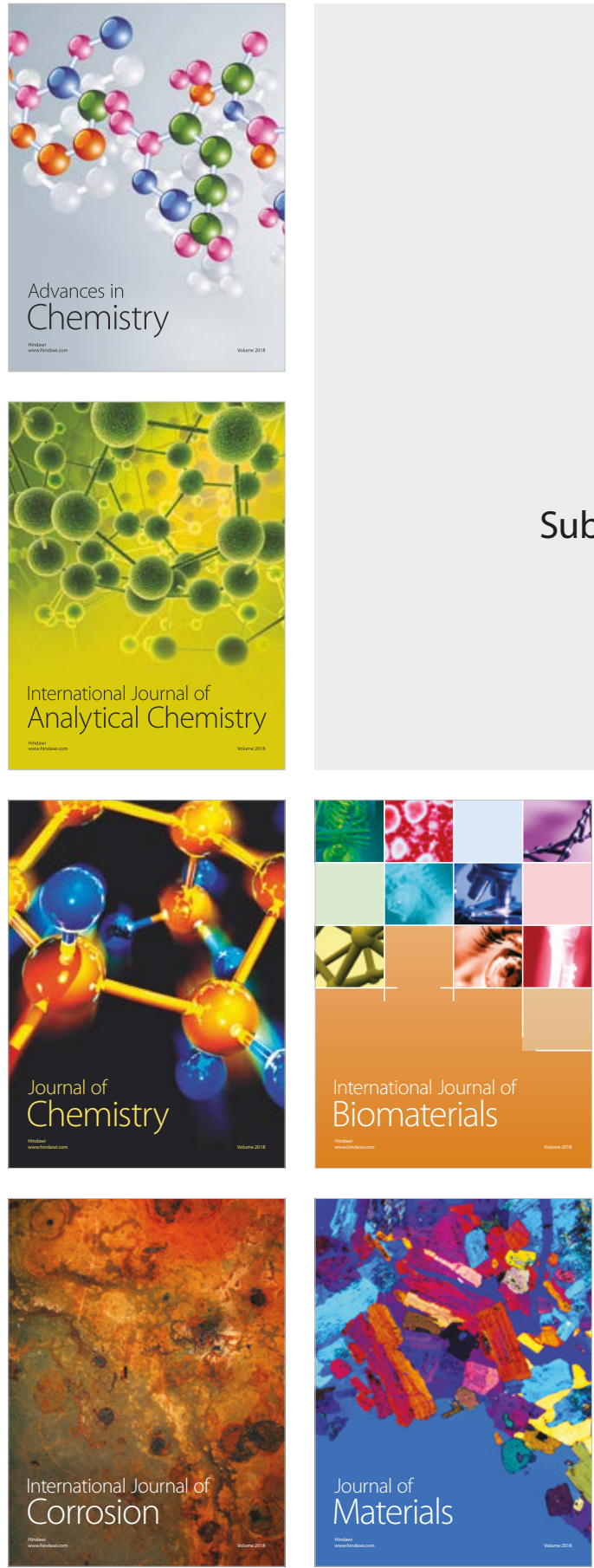

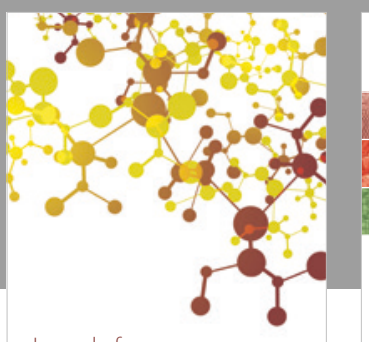

Journal of

Applied Chemistry
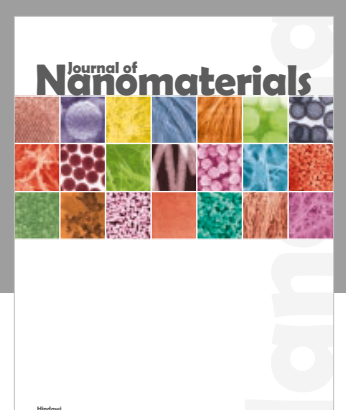

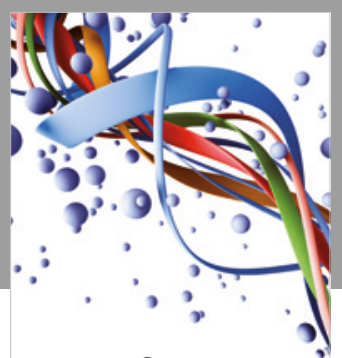

Scientifica

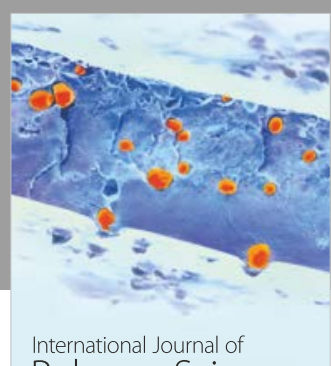

Polymer Science

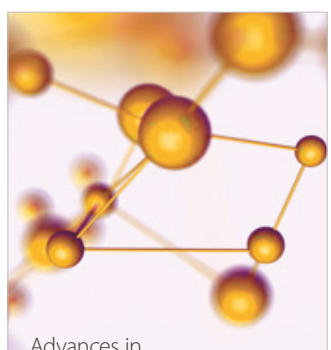

Physical Chemistry
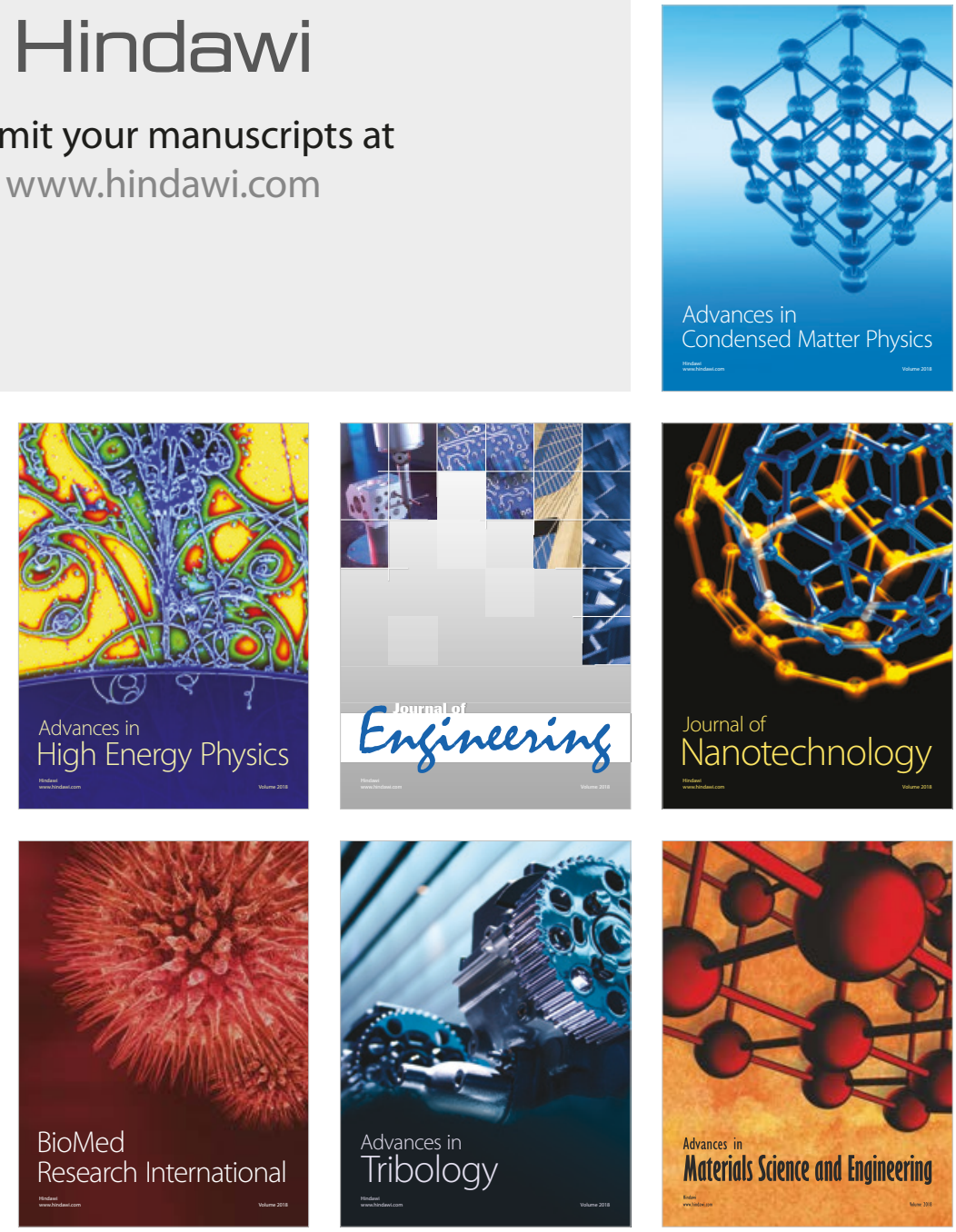